\title{
A supervised discriminant subspaces-based ensemble learning for binary classification
}

\author{
Hamidullah Binol", Huseyin Cukur and Abdullah Bal \\ Department of Electronics and Communication Engineering, Yildiz Technical University, Turkey
}

Received: 20-August-2016; Revised: 27-September-2016; Accepted: 28-September-2016

(C)2016 ACCENTS

\begin{abstract}
To enable feature extraction and reduction in pattern recognition applications, discriminant subspace analysis-based algorithms are used. Among the better-known discriminant subspace techniques for two-pattern recognition is the Fukunaga-Koontz transform (FKT). This technique has been modified to a non-linear version with the aid of kernel machines. This has enabled an increase in its non-linear discrimination ability, apart from securing higher statistics of data. The performance of kernel FKT (KFKT) is, however, dependent on the choice of suitable kernels and their inherent parameters. The aim of this paper is to ascertain the difficulties of ensemble learning with a finite set of base kernels on FKT subspaces. The study presents a new approach to tackling the issues of multiple kernel learning (MKL) on FKT. For this, a better kernel function is designed by either linearly or non-linearly combining numerous pre-chosen kernels into the algorithm. KFKTs were used with sub-kernel learners with a diverse set of kernels, each with different parameters. Weighted and unweighted fusions were employed in order to combine the predictions of sub-learners. The eventual results proved that the ensemble of KFKTs was far better than the single KFKTs as far as classification performance went.
\end{abstract}

\section{Keywords}

Classification, Ensemble learning, Fukunaga-Koontz transform, Multiple kernel learning.

\section{Introduction}

A technique, fairly similar to the Karhunen-Loève transform, is the Fukunaga-Koontz transform (FKT); this effective discrimination technique can be utilized where two-class classification issues crop up enabling retrieval of second-order relations of Gaussian distributed data $[1,2]$. Implementation of this technique has led to diverse results such as identification and ensuing tracking of targets [3, 4], recognition of faces [2], and obtaining hyperspectral images [5].

Perusal of existing work in the field over the past few decades reveals that kernel-based learning techniques have often been employed to extract statistics of nonGaussian data. For example, in the work of Liu et al. the kernel version of FKT (KFKT or kernel FKT) has been used to prove the presence of small targets in forward-looking infrared images [6].

*Author for correspondence

This research was supported by a grant from the scientific and technological research council of Turkey (TUBITAK112E207).

209
KFKT has also proved to be immensely helpful in the field of face detection and recognition [7], helping to resolve difficulties arising out of hyperspectral image classification issues $[8,9]$ as well as the dimensionality reduction of hyperspectral data [10]. Another new technique has been put forward by Binol et al. that suggest employing differential evolution algorithm-based kernel parameter selection technique for radial basis function (RBF) kernel within KFKT [11]. What makes it different from the other one is the lack of kernel evaluation and the selection method for FKT.

This paper highlights the multiple kernel learning (MKL) problems within KFKT which have its roots in the ensemble learning (EL) approach [12]. EL is an approach that works through an amalgam of several classifiers/experts. In a scenario where there is a base classifier accompanied by a set of kernels and each of these kernel-based classifiers are termed a sub-classifier, a correlation can be established between EL and MKL. In fact, as is evident from [13], it was proved that MKL is a characteristic EL. This research paper employs the fundamental premise of EL to resolve the inherent problem of 
MKL within KFKT along with using the proposed algorithm for binary classification issues. According to this technique, a kernel with different parameters is chosen. These different kernel parameters in a training data lead to a prediction result of each KFKT which, in turn, are combined with various weighted and unweighted combination processes. For the weighted processes, the weight assignment is based on the preciseness of classification of each classifier on training data. Four data sets from UPI were sourced to conduct the proposed experiments [14].

\section{Overview of kernel Fukunaga-Koontz transform}

The FKT [1] is a unique technique that selects features, in the sense that if one specific feature is selected through this technique, it provides information only about that class and gives minimal information about the other class. The inherent second-order data correlations in FKT do not provide optimum performance and this has led to many researchers in this field to employ kernel machines to enable use of FKT in its non-linear forms [6, 7]. To put it simply, with the help of a mapping scheme $\phi$ [15], the input data $\boldsymbol{x} \in \mathbb{R}^{d}$ is entered into highdimensional feature space, $\{\phi(\boldsymbol{x})\} \in \mathbb{F}$.

In such a scenario, the dot products in $F$, that is, $\left\langle\phi\left(\boldsymbol{x}_{i}\right), \phi\left(\boldsymbol{x}_{j}\right)\right\rangle$, can be assessed through kernel function, that is, $K\left(\boldsymbol{x}_{i}, \boldsymbol{x}_{j}\right)=\left\langle\phi\left(\boldsymbol{x}_{i}\right), \phi\left(\boldsymbol{x}_{j}\right)\right\rangle$, which is a positive definite function. The aforementioned kernel-based learning is described in detail along with its basic theory in [16]. Summary of data sets are shown in Table 1. For this paper, two widely used kernel functions with one free parameter are employed. These functions are Gaussian radial basis function (RBF) and spherical kernel (Table 2). To clarify, vectors of two classes, $\mathbf{C}_{1}=\left[\boldsymbol{a}_{1}, \boldsymbol{a}_{2}, \ldots, \boldsymbol{a}_{M}\right]$ which is positive and $\left[\boldsymbol{b}_{1}, \boldsymbol{b}_{2}, \ldots, \boldsymbol{b}_{M}\right]$ that is negative, are centered. The mapped versions $\mathbf{C}_{1}^{\phi}$ and $\mathbf{C}_{2}^{\phi}$ can be considered the input data. In such a situation, the resultant covariance matrices, termed the kernel matrices, can be $\boldsymbol{\Sigma}_{1}=\mathbf{C}_{1}^{\phi} \mathbf{C}_{1}^{\phi^{\top}}$ and $\boldsymbol{\Sigma}_{2}=\mathbf{C}_{2}^{\phi} \mathbf{C}_{2}^{\phi^{\top}}$. The elements of these kernel matrices can be computed by using any of the kernel functions such as $\Sigma(i, j)=$ $K\left(\boldsymbol{x}_{i}, \boldsymbol{x}_{j}\right)$. The Eigen-decomposition of the sum of training kernel matrices can be put down as shown ahead

$$
\boldsymbol{\Sigma}=\boldsymbol{\Sigma}_{1}+\boldsymbol{\Sigma}_{2}=\boldsymbol{V} \boldsymbol{\Lambda} \boldsymbol{V}^{\top}
$$

Once the transform operator $\mathbf{P}=\mathbf{V} \boldsymbol{\Lambda}^{-1 / 2}$ is formulated, we can assume

$$
\boldsymbol{P}^{\top} \boldsymbol{\Sigma} \boldsymbol{P}=\boldsymbol{I}
$$

where $\mathbf{C}_{1}^{\phi}$ and $\mathbf{C}_{2}^{\phi}$ are transformed using $\mathbf{P}$, that is, $\widehat{\mathbf{C}}_{1}\left(\hat{\mathbf{C}}_{1}=\mathbf{P}^{\top} \mathbf{C}_{1}^{\phi}\right)$ and $\hat{\mathbf{C}}_{2}\left(\hat{\mathbf{C}}_{2}=\mathbf{P}^{\top} \mathbf{C}_{2}^{\phi}\right)$. Now the altered $\hat{\mathbf{C}}_{1}$ and $\hat{\mathbf{C}}_{2}$ constitute the new covariance matrices:

$$
\begin{aligned}
& \widehat{\boldsymbol{\Sigma}}_{1}=\widehat{\boldsymbol{C}}_{1} \widehat{\boldsymbol{C}}_{1}^{\top}=\boldsymbol{P}^{\top} \boldsymbol{C}_{1}^{\phi} \boldsymbol{C}_{1}^{\phi^{\top}} \boldsymbol{P}=\boldsymbol{P}^{\top} \boldsymbol{\Sigma}_{1} \boldsymbol{P} \\
& \widehat{\boldsymbol{\Sigma}}_{2}=\widehat{\boldsymbol{C}}_{2} \widehat{\boldsymbol{C}}_{2}^{\top}=\boldsymbol{P}^{\top} \boldsymbol{C}_{2}^{\phi} \boldsymbol{C}_{2}^{\phi^{\top}} \boldsymbol{P}=\boldsymbol{P}^{\top} \boldsymbol{\Sigma}_{2} \boldsymbol{P}
\end{aligned}
$$

The key point of KFKT can be deduced from (2) and (3) given earlier as

$$
\widehat{\boldsymbol{\Sigma}}=\widehat{\boldsymbol{\Sigma}}_{1}+\widehat{\boldsymbol{\Sigma}}_{2}=\boldsymbol{P}^{\top}\left(\boldsymbol{\Sigma}_{1}+\boldsymbol{\Sigma}_{2}\right) \boldsymbol{P}=\boldsymbol{I}
$$

It can be assumed that if $\boldsymbol{v}_{\boldsymbol{i}}$ is the eigenvector of $\widehat{\boldsymbol{\Sigma}}_{1}$ that correlates to Eigen value $\lambda_{i}$, then $\left(1-\lambda_{i}\right)$ is likely to be the eigenvalue of $\widehat{\boldsymbol{\Sigma}}_{2}$ corresponding to the same eigenvector. Further, the quadratic correlation filter (QCF) is used to ascertain the class of test vector $\mathbf{z}$ [17]. The output of the optimal coefficient matrix for QCF for the test vector will be greater for $\boldsymbol{z} \in \mathbf{C}_{1}$ (positive class) and smaller for $\boldsymbol{z} \in \mathbf{C}_{2}$ (negative class). It is observed that matrix elements of $\mathbf{V}$ are eigenvectors associated with the eigenvalues of $\widehat{\boldsymbol{\Sigma}}_{1}$ and organised in descending order as $\lambda_{1} \geq$ $\lambda_{2} \geq \ldots \geq \lambda_{M} \geq 0 . \quad \mathbf{V}_{1} \quad$ denotes the first $m_{1}$ eigenvectors and belongs to the positive class and $\mathbf{V}_{2}$ denoting the rest of the eigenvectors, represents the negative class. The equation ahead gives the output of the classification system

$$
y_{\text {out }}=\boldsymbol{R}_{1}^{\top} \boldsymbol{R}_{1}-\boldsymbol{R}_{2}^{\top} \boldsymbol{R}_{2}
$$

Where $\mathbf{R}_{1}$ is $\mathbf{V}_{1}^{\top} \widehat{\boldsymbol{Z}}^{\phi}, \mathbf{R}_{2}$ is $\mathbf{V}_{2}^{\top} \widehat{\boldsymbol{Z}}^{\phi}$, and $\hat{\boldsymbol{Z}}^{\phi}$ is the transformed version of $\boldsymbol{z}^{\phi}$ with $\mathbf{P}$. When the mapping function is avoided and computation is used on its own, $\boldsymbol{\Sigma}_{1}$ is centralized [18] by

$$
\widetilde{\boldsymbol{\Sigma}}_{1}=\boldsymbol{\Sigma}_{1}-\boldsymbol{I}_{M} \boldsymbol{\Sigma}_{1}-\boldsymbol{\Sigma}_{1} \boldsymbol{I}_{M}+\boldsymbol{I}_{M} \boldsymbol{\Sigma}_{1} \boldsymbol{I}_{M}
$$

Where $\mathbf{I}_{M}$ is the $M \times M$ matrix which has the same elements of $1 / \mathrm{M}$.

The eigenvectors of $\widetilde{\boldsymbol{\Sigma}}_{1},\left\{\boldsymbol{\theta}_{i}\right\}$, that are related to the $m_{1}$ give the largest eigenvalues $\lambda_{1} \geq \lambda_{2} \geq \ldots \geq \lambda_{m_{1}}$. The $i$-th feature vector $f_{i}$ of the testing vector $\boldsymbol{z}$ is determined as

$$
f_{i}=\frac{1}{\sqrt{\lambda_{i}}} \boldsymbol{\theta}_{i}^{\top}\left[K\left(\boldsymbol{a}_{1}, \mathbf{z}\right), K\left(\boldsymbol{a}_{2}, \mathbf{z}\right), \ldots, K\left(\boldsymbol{a}_{M}, \boldsymbol{z}\right)\right]
$$

The feature vector $\boldsymbol{f}=\left[f_{1}, f_{2}, \ldots, f_{m_{1}}\right]$ is modified, $\widehat{\boldsymbol{f}}=\mathbf{P}^{\top} \boldsymbol{f}$, and projected onto the subspaces

$$
\boldsymbol{R}_{1}=\boldsymbol{V}_{1}^{\top} \hat{\boldsymbol{f}} \text { and } \boldsymbol{R}_{2}=\boldsymbol{V}_{2}^{\top} \widehat{\boldsymbol{f}}
$$

Eventually, the (5) can be redrafted as $y_{\text {out }}=$ $\mathbf{R}_{1}^{\top} \mathbf{R}_{1}-\mathbf{R}_{2}^{\top} \mathbf{R}_{2}$. 


\section{Constructing a KFKT ensemble}

Ensemble learning (EL) is a statistical mode of learning that makes use of numerous learning algorithms. This combined method leads to better and enhanced performance. Many sub-classifiers with their own precise fusion rules are combined to lead to a better result [19]. Studies in this field reveal the presence of multiple strategies pertaining to feature, data, and classifier combinations [20].

At the classifier level, diverse classifiers (either the same or of different kinds) are directed at the same specific data. The predictions that are obtained are merged, thus leading to better classification outcomes. For this research to resolve the MKL issue, a similar combined strategy has been employed. Figure 1 depicts a general architecture of the proposed KFKT ensemble.

Fusion techniques generally used for EL lead to combined results called decision level fusion. Each class is given winner labels before being confirmed. Further, the linear fusing methods are of two kinds, unweighted or majority, and weighted voting. The former is simpler as all voters have the same weight. The unweighted average of sub-KKFT's results (as shown in (9)) is then taken to calculate the decision for the $i$-th sample (because of the two labels, +1 and -1 for positive and negative class).

$f_{i, u w v}=\left\{\begin{array}{cc}-1, & \frac{1}{M} \sum_{k=1}^{M} f_{i, K F K T}^{k}<0 \\ 1, & \frac{1}{M} \sum_{i=1}^{M} f_{i, K F K T}^{k} \geq 0\end{array}\right.$

In (9), $M$ is the number of sub-classifiers and $f_{\mathrm{i}, \mathrm{uw} v}($.$) is the final decision label for i$-th sample. Even though the majority or unweighted method is the easiest technique to combine, it may not provide the perfect results. Unlike in weighted voting, where each voter has samples of different weights and the final decision is taken accordingly (10).

$$
f_{i, w v}=\left\{\begin{array}{cc}
-1, & \frac{1}{M} \sum_{k=1}^{M} w_{k} f_{i, K F K T}^{k}<0 \\
1, & \frac{1}{M} \sum_{k=1}^{M} w_{k} f_{i, K F K T}^{k} \geq 0
\end{array}\right.
$$

Where $w_{k}$ is the weight for each classifier. A crucial issue inherent in the weighted combination technique is related to the calculation of the ideal weight of each base classifier [21]. In this technique, the weight is generally determined based on the proportion to the classification accuracies of base classifiers on training data [22]. The formulation employed to decide the weights in classifier fusion for the purposes of this study is given ahead

$$
w_{k}=\frac{1}{N} \sum_{k=1}^{N}\left(f_{D V} \times f_{k}\right)
$$

Here $N$ refers to the number of samples in training data, $f_{D V}$ denotes the true labels (ground-truth), and $f_{k}$ refers to a sub-classifier's (or a sub-kernel's) predictions.

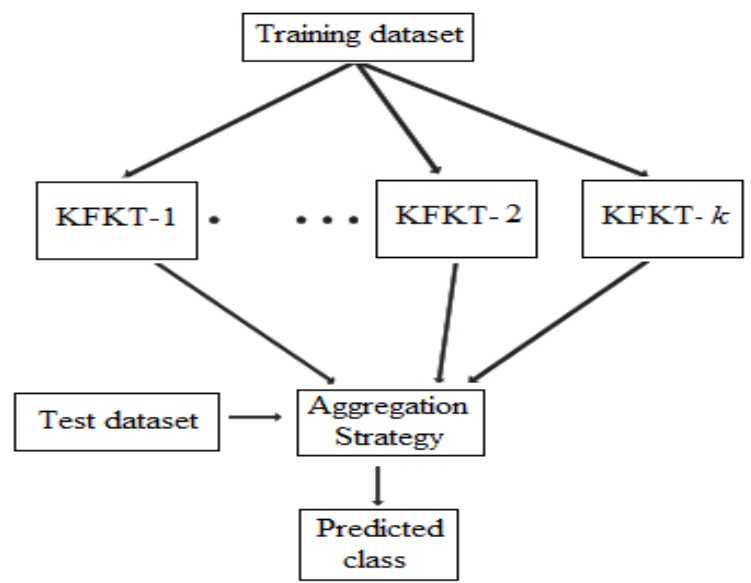

Figure 1 A general architecture of KFKT ensemble

\section{Experiments}

This segment of the study gives facts about the data used for the research, the norms used to assess the performance, and the results of the experiments performed.

\subsection{Test data}

This section evaluates the functioning of ensemble KFKT algorithms used for binary classification problems. Four data sets available in the UCI repository were chosen arbitrarily to conduct the tasks, the results of which are shown in Table 1.

\subsection{Performance evaluation}

The evaluation of classification performance is generally undertaken through classification accuracy and Kappa coefficient [23]. The classification accuracy of binary classification is formulated as

$$
A c c=(T P+T N) /(P+N)
$$

Where $T P$ denotes the true positives and $T N$ the true negatives about a classifier.

Table 1 Summary of data sets

\begin{tabular}{llll}
\hline Name & \# Classes & \# Instances & \# Attributes \\
\hline Ionosphere & 2 & 351 & 33 \\
Wisconsin & 2 & 683 & 9 \\
$\begin{array}{l}\text { Breast Cancer } \\
\text { (Original) }\end{array}$ & & & \\
Sonar & 2 & 208 & 59 \\
Skin & 2 & 245057 & 3 \\
\hline
\end{tabular}


Hamidullah Binol et al.

Table 2 Employed kernel functions

\begin{tabular}{ll}
\hline Name & Formula \\
\hline RBF & $K_{R B F}(\boldsymbol{x}, \boldsymbol{y})=\exp \left(-\|\boldsymbol{x}-\boldsymbol{y}\|^{2} / 2 \sigma^{2}\right)$ \\
$\begin{array}{l}\text { Spheric } \\
\text { al }\end{array}$ & $K_{\text {Spherical }}(\boldsymbol{x}, \boldsymbol{y})=1-3 / 2 \frac{\|\boldsymbol{x}-\boldsymbol{y}\|}{\sigma}$ \\
& $+1 / 2\left(\frac{\|\boldsymbol{x}-\boldsymbol{y}\|}{\sigma}\right)^{3}$ \\
\hline
\end{tabular}

In (12), the number of data samples in the set is considered the denominator. The Kappa coefficient, shown ahead, evaluates the accuracy of the system with the accuracy of a random system

$$
\text { kappa }=\frac{(\text { Acc-Random } A c c)}{(1-\text { Random } A c c)}
$$

Here, random accuracy (Random Acc) refers to the total of the products of reference likelihood and result likelihood for each specific class. Kappa, in this case, is

$$
\frac{[(T N+F P) \times(T N+F N)+(F N+T P) \times(F P+T P)]}{[(P+N) \times(P+N)]}
$$

Where $F N$ is the number of false negatives, and $F P$ is the number of false positives.

\subsection{Experimental results}

For each data set, RBF and spherical kernels (see Table 2) with 21 different bandwidths are utilized separately to construct sub-kernels. The bandwidths for $\mathrm{RBF}$ kernel are $\left\{\ldots, \sigma_{0}-0.2, \sigma_{0}-0.1, \sigma_{0}, \sigma_{0}+\right.$ $\left.0.1, \sigma_{0}+0.2, \ldots\right\}$, and $\left\{\sigma_{0}, \sigma_{0}+0.2, \sigma_{0}+0.4, \ldots\right\}$ for spherical kernel. The central parameter $\sigma_{0}$ of both kernels for each training data is set

$\sigma_{0}=\sqrt{\sum_{N}\left\|x^{k}-\bar{x}\right\|^{2} / N}$

Where $\bar{x}$ is the centroid of the total $N$ training data.

The training samples for each trial are chosen arbitrarily with constant training sample percentages given. For instance, for skin data, $0.1 \%$ is the chosen sample for training, whereas for sonar, ionosphere, or Wisconsin breast cancer data (Original; WBCO), the percentage is set at $10 \%$. The processes are repeated 10 times and their mean values $(\mu)$ and standard deviations (STD) of metrics are calculated. The best scores for each data set in Table 3 and Table 4 are highlighted in boldface font.

Table 3 Accuracy (Acc) (\%) and Kappa statistic $(\kappa)(\%)$ for RBF kernel with different algorithms over 10

\begin{tabular}{|c|c|c|c|c|c|c|c|c|}
\hline \multirow[t]{3}{*}{ Data set } & \multirow{3}{*}{$\begin{array}{l}\text { Percentage } \\
\text { of training } \\
\text { samples }\end{array}$} & & \multirow{2}{*}{\multicolumn{2}{|c|}{ KFKT }} & \multicolumn{4}{|c|}{ E-KFKT } \\
\hline & & & & & \multicolumn{2}{|c|}{ Majority voting } & \multicolumn{2}{|c|}{ Weighted voting } \\
\hline & & & Acc & $\kappa$ & Acc & $\kappa$ & Acc & $\kappa$ \\
\hline \multirow[t]{2}{*}{ Ionosphere } & 10 & $\mu$ & 73.84 & 51.35 & 80.88 & 61.98 & 83.70 & 66.91 \\
\hline & & std & 6.15 & 9.90 & 6.99 & 12.02 & 4.99 & 8.85 \\
\hline \multirow[t]{2}{*}{ WBCO } & 10 & $\mu$ & 63.15 & 40.29 & 58.18 & 33.00 & 81.35 & 67.07 \\
\hline & & std & 18.22 & 27.14 & 28.23 & 42.32 & 21.92 & 34.63 \\
\hline \multirow[t]{2}{*}{ Sonar } & 10 & $\mu$ & 57.93 & 10.87 & 57.93 & 10.64 & 58.70 & 12.24 \\
\hline & & std & 1.34 & 2.86 & 1.23 & 2.64 & 1.12 & 2.54 \\
\hline \multirow[t]{2}{*}{ Skin } & 0.1 & $\mu$ & 78.20 & 24.59 & 79.80 & 4.68 & 98.86 & 96.61 \\
\hline & & std & 2.68 & 1.23 & 0.69 & 5.56 & 0.35 & 1.01 \\
\hline
\end{tabular}
independent runs.

Table 4 Accuracy (Acc) $(\%)$ and Kappa statistic $(\kappa)(\%)$ for spherical kernel with different algorithms over 10

\begin{tabular}{|c|c|c|c|c|c|c|c|c|}
\hline \multirow[t]{3}{*}{ Data set } & \multirow{3}{*}{$\begin{array}{l}\text { Percentage } \\
\text { of training } \\
\text { samples }\end{array}$} & & \multicolumn{2}{|l|}{ KFKT } & \multicolumn{4}{|c|}{ E-KFKT } \\
\hline & & & \multirow[b]{2}{*}{ Acc } & \multirow[b]{2}{*}{$\kappa$} & \multicolumn{2}{|c|}{ Majority voting } & \multicolumn{2}{|c|}{ Weighted voting } \\
\hline & & & & & Acc & $\kappa$ & Acc & $\kappa$ \\
\hline \multirow[t]{2}{*}{ Ionosphere } & 10 & $\mu$ & 48.77 & 15.56 & 53.42 & 21.19 & $\mathbf{5 7 . 2 1}$ & 26.16 \\
\hline & & std & 1.77 & 2.46 & 3.78 & 5.03 & 2.48 & 3.61 \\
\hline \multirow[t]{2}{*}{ WBCO } & 10 & $\mu$ & 67.82 & 45.74 & 78.57 & 59.76 & 84.57 & 69.51 \\
\hline & & std & 5.89 & 9.35 & 11.50 & 17.33 & 5.51 & 9.85 \\
\hline \multirow[t]{2}{*}{ Sonar } & 10 & $\mu$ & 56.88 & 8.04 & 57.31 & 8.97 & 57.64 & 9.73 \\
\hline & & std & 0.65 & 1.47 & 0.93 & 2.11 & 0.27 & 0.62 \\
\hline \multirow[t]{2}{*}{ Skin } & 0.1 & $\mu$ & 70.06 & 40.71 & 69.85 & 40.30 & 80.53 & 61.31 \\
\hline & & std & 3.05 & 6.00 & 5.73 & 11.21 & 5.37 & 10.60 \\
\hline
\end{tabular}
independent runs.

Table 3 presents the results of all techniques used for RBF kernels. The bold fonts depict the winners, the 212 majority or unweighted voting is shown to improve the performance in the skin and the ionosphere. 
However, the performance goes down in the WBCO. As for the sonar set, there is no significant difference between majority voting based E-KFKT and single KFKT, as far as Kappa and accuracy are concerned. Overall, it proves that weighted voting E-KFKT leads to much better classification performance for all data sets chosen.

Table 4 presents the results obtained through various methods for spherical kernels. Here, E-KFKT with majority voting is seen to get better performance results than single KFKT, except in the skin. All data sets have better classification accuracy with E-KFKT with weighted voting.

\section{Conclusion}

This paper presents an ensemble learning-based multiple kernel FKT in order to enhance the performance of a KFKT classifier. For this purpose, pre-defined kernel sets were developed by using two different kernels with different parameters. The linear combination of these KFKTs that resulted through the candidate kernels were obtained through unweighted and weighted voting methods of EL. The experiments conducted showed the impact of the suggested technique on MKL in KFKT.

Further research will use selective EL to improve this strategy. The numbers and multiplicity of kernels could also form an important aspect of future research in the field. The work undertaken in this study to classify binary patterns could also be employed for multi-class tasks.

\section{Acknowledgment}

None.

\section{Conflicts of interest}

The authors have no conflicts of interest to declare.

\section{References}

[1] Fukunaga K, Koontz WL. Application of the Karhunen-Loeve expansion to feature selection and ordering. IEEE Transactions on Computers. 1970; 19(4):311-8.

[2] Yang MH, Kriegman DJ, Ahuja N. Detecting faces in images: a survey. IEEE Transactions on Pattern Analysis and Machine Intelligence. 2002; 24(1):34-58.

[3] Bal A, Alam MS. Quadratic correlation filter based target tracking in FLIR image sequences. In optics \& photonics 2005. International Society for Optics and Photonics.

[4] Mahalanobis A, Muise RR, Stanfill SR, Van Nevel AL. Design and application of quadratic correlation filters for target detection. IEEE Transactions on
Aerospace and Electronic Systems.2004; 40(3):83750.

[5] Ochilov S, Alam MS, Bal A. Fukunaga-Koontz transform based dimensionality reduction for hyperspectral imagery. In defense and security symposium 2006. International Society for Optics and Photonics.

[6] Liu RM, Liu EQ, Yang J, Zhang TH, Wang FL. Infrared small target detection with kernel FukunagaKoontz transform. Measurement Science and Technology. 2007; 18(9):3025.

[7] Li YH, Savvides M. Kernel Fukunaga-Koontz transform subspaces for enhanced face recognition. In 2007 IEEE conference on computer vision and pattern recognition 2007 (pp. 1-8). IEEE.

[8] Dinç S, Bal A. Hyperspectral image classification using kernel Fukunaga-Koontz transform. Mathematical Problems in Engineering.2013:1-7.

[9] Binol H, Bilgin G, Dinc S, Bal A. Kernel FukunagaKoontz transform subspaces for classification of hyperspectral images with small sample sizes. IEEE Geoscience and Remote Sensing Letters. 2015; 12(6):1287-91.

[10] Binol H, Ochilov S, Alam MS, Bal A. Target oriented dimensionality reduction of hyperspectral data by kernel Fukunaga-Koontz transform. Optics and Lasers in Engineering. 2016.

[11] Binol H, Bal A, Cukur H. Differential evolution algorithm-based kernel parameter selection for Fukunaga-Koontz transform subspaces construction. In SPIE remote sensing 2015. International Society for Optics and Photonics.

[12] Bach FR, Lanckriet GR, Jordan MI. Multiple kernel learning, conic duality, and the SMO algorithm. In proceedings of the twenty-first international conference on machine learning 2004 (p. 6). ACM.

[13] Sun T, Jiao L, Liu F, Wang S, Feng J. Selective multiple kernel learning for classification with ensemble strategy. Pattern Recognition. 2013; 46(11):3081-90.

[14] Lichman M. UCI machine learning repository. School Information Computer Science, University of California, Irvine, CA, USA. http://archive.ics.uci.edu/ml. Accessed 10 June 2016.

[15] Aizerman A, Braverman EM, Rozoner LI. Theoretical foundations of the potential function method in pattern recognition learning. Automation and Remote control. 1964; 25:821-37.

[16] Shawe-Taylor J, Cristianini N. Kernel methods for pattern analysis. Cambridge university press; 2004.

[17] Huo X, Elad M, Flesia AG, Muise RR, Stanfill SR, Friedman $\mathrm{J}$, et al. Optimal reduced-rank quadratic classifiers using the Fukunaga-Koontz transform with applications to automated target recognition. In Aero Sense 2003 (pp. 59-72). International Society for Optics and Photonics.

[18] Yang J, Frangi AF, Yang JY, Zhang D, Jin Z. KPCA plus LDA: a complete kernel fisher discriminant framework for feature extraction and recognition. 
IEEE Transactions on Pattern Analysis and Machine Intelligence. 2005; 27(2):230-44.

[19] Kuncheva LI, Whitaker CJ. Measures of diversity in classifier ensembles and their relationship with the ensemble accuracy. Machine Learning. 2003; 51(2):181-207.

[20] Bryll R, Gutierrez-Osuna R, Quek F. Attribute bagging: improving accuracy of classifier ensembles by using random feature subsets. Pattern Recognition. 2003; 36(6):1291-302.

[21] Lee WJ, Verzakov S, Duin RP. Kernel combination versus classifier combination. In international workshop on multiple classifier systems 2007 (pp. 2231). Springer Berlin Heidelberg.

[22] Kim HC, Pang S, Je HM, Kim D, Bang SY. Support vector machine ensemble with bagging. In pattern recognition with support vector machines 2002 (pp. 397-408). Springer Berlin Heidelberg.

[23] Richards JA. Remote sensing digital image analysis. Springer; 1999.

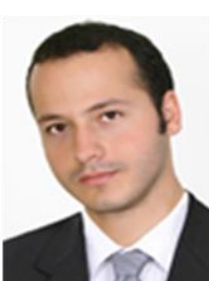

Hamidullah Binol is a research assistant at the Yildiz Technical University. He received the B.S. degree in Telecommunications Engineering from the Istanbul Technical University in 2010, and the M.S. degree in Electronics and Communications Engineering from the Yildiz Technical University in 2012. He is currently a Ph.D. candidate in the Electronics and Communications Engineering Department at the same university. His current research interests include hyperspectral data processing, machine learning applications in remote sensing, kernel-based methods in machine learning, and pattern recognition.

Email: hbinol@yildiz.edu.tr

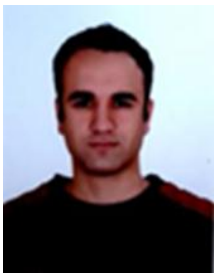

Huseyin Cukur is a research assistant at the Yildiz Technical University. $\mathrm{He}$ received the B.S. degree from the Department of Electronics Engineering from the Istanbul Technical University, Istanbul, Turkey, in 2012, and the M.S. degree from the Department of Electronics and Communications Engineering from the Yildiz Technical University, Istanbul, Turkey, in 2016. He is currently working toward the Ph.D. degree in the Department of Electronics and Communications Engineering, Yildiz Technical University, Istanbul, Turkey. His research interests include pattern recognition and machine learning.

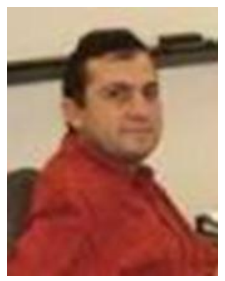

Abdullah Bal received his Ph.D. degree in Electrical Engineering from Yildiz Technical University, Istanbul, Turkey. Currently, he is a Professor at Electronics and Communication Engineering Department of Yildiz Technical University. His research interests include digital/optical signal and image processing, remote sensing, pattern recognition, artificial neural networks, machine learning, wavelet theory, and data/decision fusion. 